\title{
Entrenamiento y certificación en endoscopia ginecológica
}

\author{
Ramiro Argüello Argüello*
}

\begin{abstract}
RESUMEN: El desarrollo de la Endoscopia ginecológica en los últimos años ha hecho que los procedinientos operatorios laparohisteroscópicos sean cada vez más frecuentes, y en Colombia un $60 \%$ a $70 \%$ de los procedimientos que antiguamente se realizaban exclusivamente mediante Laparotomía se pueden hacer ahora mediante Cirugía Laparoscópica. La Sociedad Colombiana de Obstetricia y Ginecología (S.C.O.G.) ha trabajado desde 1.995 en la Reglamentación de la Endoscopia Ginecológica en Colombia y con este propósito creó la Comisión de Certificación en Endoscopia Ginecológica. Se presentan los logros alcanzados por esta Comisión en los últimos años. Se presenta la clasificación actual de los procedimientos Endoscópicos Ginecológicos en Colombia y los requisitos exigidos por la S.C.O.G. para la certificación de los niveles básico, avanzado y experto. Finalmente se invita a los ginecólogos interesados en el tema a ponerse en contacto con la Sede Departamental o Nacional de la S.C.O.G. con el fin de ampliar la información presentada.
\end{abstract}

PALABRAS CLAVES: Laparoscopia, endoscopia ginecológica, entrenamiento, certificación, acreditación.

SUMMARY: The development of Endoscopic Surgery in gynecology, in recent years, has made the laparo-histeroscopic surgery more frequent every day, and in Colombia about $60 \%$ to $70 \%$ of the procedures that formerly was made only by laparotomy now can be performed by Laparoscopy. The Sociedad Colombiana de Obstetricia y Ginecología (S.C.O.G.) has been working in the regulations of Gynecologic Endoscopy in Colombia and with this purpose created the Comisión de Certificación en Endoscopia Ginecológica. The goals fulfilled by this Committee are presented. An actual classification of Gynecologic Laparoscopic procedures in Colombia is also presented as well as the S.C.O.G.'s requirements to grant certification in basic, advanced and expert level. Finally, an invitation is made to the gynecologist with an special interest about this topic, to put in contact with the local o national headquarters of the S.C.O.G. in order to obtain more information.

KEY WORDS: Laparoscopy, gynecological endoscopy, training, certification, credentialling.

\section{Introducción}

Desde 1987, cuando Phillippe Mauriat realizó la primera Colecistectomía por Laparoscopia en Lyon, Francia (1), los procedimientos quirúrgicos realizados mediante técnicas que podemos llamar de videoendoscopia, antes llamados de Invasión Mínima, se han venido multiplicando día a día en todas las especialidades (2). La Cirugía Ginecológica ha sido uno de los campos en que esta tendencia se ha mostrado de manera mas clara (3), y en Colombia ya podemos afirmar que aproximadamente un $60 \%$ a $70 \%$ de los procedimientos que antiguamente se realizaban exclusivamente mediante laparotomía se pueden hacer ahora mediante Cirugía Laparoscópica. Para confirmar lo anterior basta revisar los Manuales de Procedimientos y Tarifas más usados en nuestro medio (4-5).

La Sociedad Colombiana de Obstetricia y Ginecología (S.C.O.G.) consciente de este cambio revolucionario en la Cirugía Ginecológica, empezó a trabajar desde 1995 en la Reglamentación de la Endoscopia Ginecológica en Colombia, buscando brindar parámetros de control que permitieran a los ginecólogos, a las instituciones y a los organismos de salud evaluar los servicios de laparo-histeroscopia que

Coordinador de la Comisión de Certificación en Endoscopia Ginecológica de la Sociedad Colombiana de Obstetricia y Ginecología. Certificación de Experto en Endoscopia Ginecológica dado por la Sociedad Colombiana de Obstetricia y Ginecología. Director Científico de la Unidad de Endoscopia Ginecológica y Dolor Pélvico. Unegin Ltda están actualmente en funcionamiento y aportar directrices que sirvieran de guía a las que se inicien en el futuro.

Como aspectos positivos logrados por la S.C.O.G. en la Reglamentación de la Endoscopia Ginecológica pueden mencionarse los siguientes:

1. Realizó una clasificación de los procedimientos en Endoscopia Ginecológica teniendo en cuenta la tecnología requerida, el tiempo empleado para su realización, su nivel de complejidad y el riesgo para la paciente (Tablas No. 1 y 2), y estimó en cada procedimiento laparo-histeroscópico las Unidades de Valor Relativo (U.V.R.) correspondientes a los honorarios quirúrgicos.

2. Determinó los requisitos en cuanto dotación, equipo, instrumental y funcionamiento que, según la Comisión, brindaran en cada institución las garantías necesarias en los procedimientos de Endoscopia Ginecológica.

3. Trabajó de manera conjunta con las principales E.P.S. del país con el fin de lograr la inclusión de los procedimientos endoscópicos ginecológicos en los Manuales de Contenidos y Tarifas.

4. Determinó los requisitos básicos que, según la S.C.O.G., debe llenar un programa de entrenamiento de Ginecólogos en Endoscopia Ginecológica y de los grupos o instituciones que los dicten.

5. Diseñó un modelo de Informe escrito de Endoscopia Ginecológica que permite utilizarlo en los procedimientos laparo-histeroscópicos operatorios (6). 
Tabla 1

ENDOSCOPIA GINECOLOGICA

\section{CIRUGIA GINECOLOGICA LAPAROSCOPICA DE COMPLEJIDAD BASICA}

* fulguracion de ovario uni o bilateral

* seccion de adHerencias a pared abdominal

* extraccion de d.I.U intraabdominal

* seccion y/o ligadura de ambas trompas de falopio

* tratamiEnTO DE ENDOMETRIOSIS, ESTADOS I Y II

* aspiracion de Quistes de ovario, unico o multiple, uni o bilateral

6. Inició el estudio de hojas de vida presentadas a la Sociedad Bogotana de Obstetricia y Ginecología (S.B.O.G.) en donde se solicitaba Certificación en Endoscopia Ginecológica y fijó los parámetros que permitieron otorgar dicha Certificación.

7. Inició la Certificación de ginecólogos en Endoscopia Ginecológica en Santafé de Bogotá.

8. Se redactó el primer Manual de Reglamentación en Endoscopia Ginecológica con el fin de crear un do-

Tabla 2

ENDOSCOPIA GINECOLOGICA

\section{CIRUGIA GINECOLOGICA LAPAROSCOPICA DE} COMPLEJIDAD AVANZADA

- HISTERORRAFIA

- seccion de ligamentos utero sacros

- cirugia conservadora del embarazo ECTOPICO (SALPINGOSTOMIA)

- SUSPENSION UTERINA

- MIOMECTOMÍA- MIOMAS PEDICULAdOS, UNICO O MULTIPLE

- Cistectomia (Quiste de ovario O INTRALIGAMENTARIO).

- SALPINGOLISIS Y/O OVARIOLISIS.

- SAlPingeCTOMIA, OOFORECTOMIA O SALPINGOOFORECTOMIA.

- SALPINGOPLASTIA.

- tratamiento laparoscopico endoMETRIOSIS ESTADO III Y IV.

- MiOMECTOMIA- MIOMA SUBSEROSO, UNICO O MULTIPLE.

- SALPINGONEOSTOMIA.

- NEURECTOMIA PRESACRA.

- colpopexia.

- uRETROCistopexia

- histerectomia por laparoscopia (no incluida en el I.S.S. o en el S.O.A.T.) cumento que sirviera de base para difundir la experiencia obtenida en Santafé de Bogotá.

9. Este primer Manual de Reglamentación en Endoscopia Ginecológica fue presentado a nivel nacional y discutido por comisiones en el Curso Precongreso del Congreso Nacional de la S.C.O.G. realizado en la ciudad de Medellín en 1996, y aspectos pertinentes a la reglamentación del ejercicio de la Endoscopia Ginecológica en Colombia fueron presentados y discutidos en dos Asambleas Nacionales de la FECOLSOG (ahora S.C.O.G.).

10. En Asamblea Nacional de la FECOLSOG se aprobó, mediante votación de las diferentes filiales, llevar a nivel nacional el proceso de Certificación en Endoscopia Ginecológica y posteriormente la Junta Directiva creó la Comisión de Certificación en Endoscopia Ginecológica de la S.C.O.G., encargándola de esa tarea.

Uno de los aspectos más importantes es el referente al entrenamiento y certificación de ginecólogos en Endoscopia Ginecológica. Este tema despierta interés no solo en Colombia sino en todo el mundo. Los requisitos específicos que exige la S.C.O.G. para los niveles básico, avanzado y experto se describen en la Tabla No. 3.

\section{Discusión}

Cada vez es mayor el número de procedimientos quirúrgicos ginecológicos que, gracias a la laparoscopia operatoria, se pueden realizar de manera ambulatoria (45), con los beneficios para los pacientes y la reducción de costos por todos conocidos. Este hecho acompañado de un continuo desarrollo tecnológico en equipo e instrumental y de la aparición de nuevas técnicas quirúrgicas endoscópicas hacen que para el ginecólogo en ejercicio sea indispensable recibir un entrenamiento adecuado en esta área que lo capacite para dominar las técnicas básicas y avanzadas de la Cirugía Laparo-histeroscópica moderna (7), evitando al máximo las posibles complicaciones quirúrgicas (8).

Qué se considera un entrenamiento adecuado y cuál debe ser el mecanismo mediante el cual el ginecólogo pueda demostrar su experiencia en este campo, es tema que suscita discusión en todo el mundo (9-12), y debe ser resuelto en cada país de acuerdo a sus características propias. 


\section{NIVEL BASICO}

Grupo en el cual, para efectos de certificación, se considera indispensable un mínimo de cincuenta (50) procedimientos de los clasificados en este nivel, realizados como cirujano, asistido por instructor o ginecólogo experimentado en el área, incluyendo los procedimientos histeroscópicos de Nivel Básico.

\section{NIVEL AVANZADO}

Se recomiendan como mínimo cincuenta (50) procedimientos de los clasificados en este nivel, realizados como cirujano, asistido por instructor o ginecólogo experimentado en el área, incluyendo los procedimientos histeroscópicos de Nivel Avanzado. Implica el dominio de las cirugías utilizando técnicas de video con entrenamientos dirigidos en simuladores pélvicos (Pelvic trainer) y haberse capacitado en un curso de entrenamiento que tenga el aval de la Sociedad Colombiana de Obstetricia y Ginecología y esté de acuerdo con las disposiciones de ley.

\section{NIVEL DE EXPERTO}

Debe demostrar un mínimo de cinco (5) años de práctica continua en el Nivel Avanzado; haber participado como docente en Cursos de Entrenamiento en Endoscopia Ginecológica para ginecólogos y haber contribuido con aportes importantes para el desarrollo de la Endoscopia Ginecológica en nuestro medio.

No sobra resaltar que estamos tratando de un tema en pleno desarrollo en el ámbito mundial y que continuamente recibe aportes por los descubrimientos y aplicaciones de la tecnología de punta, por no mencionar la presión de las casas comerciales. Es por eso que se requiere de un proceso de continua actualización y revisión si queremos evitar caer tanto en el estancamiento, utilizando equipo o técnicas obsoletas, como en el abuso, al intentar aplicar de manera rutinaria prácticas que deberían utilizarse únicamente bajo estrictos protocolos de investigación (13).

Con respecto a los límites de los procedimientos Endoscópicos Ginecológicos, las indicaciones y contraindicaciones para estos procedimientos, selección de pacientes, protocolos de manejo, inclusión de procedimientos nuevos o exclusión de aquellos que no han demostrado su utilidad, continúa siendo labor de las Sociedades Científicas y de las Instituciones Universitarias determinar pautas claras al respecto y difundirlas ampliamente, tanto en el medio académico como en la práctica privada de la ginecología.

\section{Conclusión}

La labor adelantada por la Comisión de Certificación en Endoscopia Ginecológica de la S.C.O.G., busca alcanzar los siguientes objetivos:

1. Lograr un consenso nacional con respecto a los requisitos mínimos de un entrenamiento adecuado en
Endoscopia Ginecológica, de tal manera que un determinado nivel de entrenamiento signifique lo mismo para todos los grupos y que sea posible homologar las certificaciones dadas entre ellas mismas y la Sociedad, y se puedan equiparar en el plano internacional.

2. Brindar un mecanismo para que los ginecólogos que ya tienen experiencia en Laparo-histeroscopia operatoria puedan demostrarla de manera adecuada.

3. Estimular a los ginecólogos recientemente egresados para que se capaciten en cursos de entrenamiento en Endoscopia Ginecológica que cumplan con los requisitos mencionados, y eviten los numerosos peligros que conlleva el intentar ser autodidacta en cirugía laparo-histeroscópica. Se debe recordar que sin un entrenamiento adecuado los resultados clínicos serán pobres y las tasas de complicaciones injustificadamente altas (11)

Esperamos que este aporte de la Sociedad Colombiana de Obstetricia y Ginecología a la Endoscopia Ginecológica en Colombia fortalezca el camino de excelencia que ya está tomando esta área de la ginecología en nuestro medio, y como Coordinador de la Comisión de Certificación en Endoscopia Ginecológica de la S.C.O.G. invitó a los ginecólogos interesados en este tema a ponerse en contacto con su sede Departamental, o con la sede en Santafé de Bogotá, con el fin de ampliar la información aquí presentada. 


\section{BIBLIOGRAFIA}

1. Gaskin TA., Isobe JH., Mathews JL., Winchester SB., Smith RJ. Laparoscopy and the General Sugeon. Surg. Clin. North. Am. 1991; 71: 1085-1097.

2. Broussard TD., Mckernan JB., Laws HL. Horizons in Endoscopic Surgery. En: Braverman MH., Tawes RL., eds. Surgical Technology International II. 1a. ed. San Francisco, California. Editado por Surgical Technology International 1993; 37-39.

3. Awwad JT., Isaacson K. Indications and Contraindications of Laparoscopy. En: Cohen SM., ed. Operative Laparoscopy and histeroscopy. 1a. ed. New York: Churchill Livingstone Inc.; 1996; 5-19.

4. Empresas Industriales y Comerciales del Estado. Instituto de Seguros Sociales. Acuerdo número 180 de 1998. Diario Oficial 25 de marzo, 1998.

5. Ministerio de Salud. Manual de Tarifas y Procedimientos. Decreto 2423 del 31 de diciembre de 1996. (S.O.A.T.).

6. Argüello R., Ferro J., Gómez P., Parra S. Informe de Endoscopia Ginecológica. Rev. Col. Ginecol. 1997; 48: 119-123.

7. Szabó Z., Hunter J., Litwin D., Berci G. Training For Advanced Laparoscopic Surgical Skills; Suturing, Knotting, and Anastomosis Techniques. En: Szabó Z., Kerstein M., Lewis J., eds. Surgical Technology International III. 1a. ed. San Francisco, California.
Editado por Surgical Technology International; 1994; 125-138.

8. Bateman B., Kolp L., Hoeger K. Complications of laparoscopy - operative and diagnostic. Fertil. Steril. 1996; 66:30-35.

9. Azziz R. Training and Certification in Operative Endoscopy. En: Azziz R., Alvarez A., eds. Practical Manual of Operative Laparoscopy and Histeroscopy. Springer Verlag. New York, inc. 1992; 217-219.

10. Airan M., Tao S. Credentialing, Privileging, Proctoring, in the Era of Laparoscopic Surgery: History of Credentialing Problems Originating with Laparoscopic Surgery. En: Szabó Z., Kerstein M., Lewis J., eds. Surgical Technology International III. 1a. ed. San Francisco, California. Editado por Surgical Technology International. 1994; 119-122.

11. Keye W. Hitting a moving target: credentialing the endoscopic surgeon. Fertil. Steril. 1994; 62: 1115-1117.

12. Levinson C. Training and credentialling. En: Gomel V., Tailor P. Eds.: Diagnostic and Operative Gynecologic Laparoscopy. San Louis, Missouri: Mosby Year Book Inc. 1995; 6-12.

13. Troidl H. Endoscopic Surgery - A Fascinating Idea Requires Responsability in Evaluation and Handling. En: Szabó Z., Kerstein M., Lewis J., eds. Surgical Technology International III. 1a. ed. San Francisco, California. Editado por Surgical Technology International. 1994; 111-117. 